ACCePted On OCt. 29, 2016

Preprint typeset using $\mathrm{L}_{\mathrm{A}} \mathrm{EX}$ style emulateapj v. 5/2/11

\title{
3.5-YEAR MONITORING OF 225 GHZ OPACITY AT THE SUMMIT OF GREENLAND
}

\author{
Satoki Matsushita $^{1}$, Keilchi Asada ${ }^{1}$, Pierre L. Martin-Cocher ${ }^{1}$, Ming-Tang Chen ${ }^{1}$, Paul T. P. Ho ${ }^{1}$, Makoto \\ Inoue $^{1}$, Patrick M. Koch ${ }^{1}$, Scott N. Paine ${ }^{2}$, David D. Turner ${ }^{3}$ \\ Accepted on Oct. 29, 2016
}

\section{ABSTRACT}

We present the 3.5-yr monitoring results of $225 \mathrm{GHz}$ opacity at the summit of the Greenland ice sheet (Greenland Summit Camp) at an altitude of $3200 \mathrm{~m}$ using a tipping radiometer. We chose this site as our submillimeter telescope (Greenland Telescope; GLT) site, because conditions are expected to have low submillimeter opacity and because its location offers favorable baselines to existing submillimeter telescopes for global-scale Very Long Baseline Interferometry (VLBI). The site shows a clear seasonal variation with the average opacity lower by a factor of two during winter. The 25\%, 50\%, and $75 \%$ quartiles of the $225 \mathrm{GHz}$ opacity during the winter months of November through April are 0.046, 0.060 , and 0.080 , respectively. For the winter quartiles of $25 \%$ and $50 \%$, the Greenland site is about 10\% - 30\% worse than the Atacama Large Millimeter/submillimeter Array (ALMA) or the South Pole sites. Estimated atmospheric transmission spectra in winter season are similar to the ALMA site at lower frequencies $(<450 \mathrm{GHz})$, which are transparent enough to perform astronomical observations almost all of the winter time with opacities $<0.5$, but $10 \%-25 \%$ higher opacities at higher frequencies $(>450 \mathrm{GHz}$ ) than those at the ALMA site. This is due to the lower altitude of the Greenland site and the resulting higher line wing opacity from pressure-broadened saturated water lines in addition to higher dry air continuum absorption at higher frequencies. Nevertheless, half of the winter time at the Greenland Summit Camp can be used for astronomical observations at frequencies between $450 \mathrm{GHz}$ and $1000 \mathrm{GHz}$ with opacities $<1.2$, and $10 \%$ of the time show $>10 \%$ transmittance in the $\mathrm{THz}(1035 \mathrm{GHz}, 1350 \mathrm{GHz}$, and $1500 \mathrm{GHz}$ ) windows. Summer season is good for observations at frequencies lower than $380 \mathrm{GHz}$. One major advantage of the Greenland Summit Camp site in winter is that there is no diurnal variation due to the polar night condition, and therefore the durations of low-opacity conditions are significantly longer than at the ALMA site. Opacities lower than 0.05 or 0.04 can continue for more than 100 hours. Such long stable opacity conditions do not occur as often even at the South Pole; it happens only for the opacity lower than 0.05. Since the opacity variation is directly related to the sky temperature (background) variation, the Greenland Summit Camp is suitable for astronomical observations that need unusually stable sky background.

Subject headings: atmospheric effects; site testing

\section{INTRODUCTION}

As various technologies for submillimeter (submm) wave observations have advanced, the prospects for very long baseline interferometry (VLBI) at submm wavelengths have become a reality. Operations at shorter wavelengths improve proportionately the spatial resolution as compared with current centimeter and millimeter VLBI observations; we anticipate that it will be possible to resolve astronomical sources in greater details, by a factor of 10 .

The demand for better angular resolution is quite strong, especially for the direct imaging of nearby supermassive black holes (SMBHs); Sagittarius A* (Sgr A*), which is located at the center of our Galaxy and therefore the nearest SMBH, has been imaged at various wavelengths using the VLBI technique. The observed size of Sgr $A^{*}$ is obviously affected by the interstellar scattering at $3 \mathrm{~mm}$ or longer wavelengths, following the $\lambda^{2}$ scattering law (e.g., Shen et al. 2005; Bower et al. 2006). This

\footnotetext{
${ }^{1}$ Academia Sinica Institute of Astronomy and Astrophysics, P.O. Box 23-141, Taipei 10617, Taiwan, R.O.C.

${ }^{2}$ Harvard-Smithsonian Center for Astrophysics, 60 Garden Street, MS-78, Cambridge, MA 02138, U.S.A.

${ }^{3}$ Global Systems Division, Earth System Research Laboratory, National Oceanic and Atmospheric Administration, 325 Broadway, Boulder, CO, 80305, U.S.A.
}

effect, however, lessens at shorter wavelengths, and the size is observed to deviate from the $\lambda^{2}$ scattering law at $1.3 \mathrm{~mm}$ (Doeleman et al. 2008). This has strongly motivated submm-VLBI observations toward nearby SMBHs to resolve and image emission from their vicinities.

Upper limits of the intrinsic sizes of SMBHs have been measured so far for Sgr A* (Doeleman et al. 2008) and the nucleus of M87 (Doeleman et al. 2012); upper limits to the sizes of both sources are about $40 \mu$ arcsec. These results indicate that much longer baselines and/or higher frequencies are needed to resolve and image the SMBHs. We, therefore, started to look for a new site to perform submm-VLBI observations with substantially longer baselines than before.

\section{SITE SELECTION}

For the site selection, we set criteria as follows:

1. Annual precipitable water vapor (PWV) of less than $3 \mathrm{~mm}$ for low submm opacity.

2. Longest possible baselines with existing submm telescopes, for obtaining the highest angular resolution (this also means that we do not consider sites that already have submm telescopes).

3. Overlapping sky coverage with the Atacama 
Large Millimeter/submillimeter Array (ALMA) to achieve the highest possible sensitivity.

4. Accessibility to the site for maintenance and operations.

We checked the satellite-based PWV data measured by the Moderate Resolution Imaging Spectroradiometer (MODIS) on the NASA Aqua and Terra satellites for potential sites with respect to the locations of the available submm telescopes. We found three potential broad regions of interest; western China and Tibet, the highest mountains of southern Alaska, and the high Arctic polar desert, including northern Canada and Greenland. The Western China and Tibet regions do not have common sky with ALMA, so they do not meet the criterion (3). The tallest peaks in Alaska (e.g., Denali, or former official name Mount McKinley) are protected or otherwise inaccessible, so they do not meet the criterion (4). The summit of the Greenland ice sheet, on the other hand, has low PWV conditions throughout the year, has a common sky coverage with ALMA, will create the longest baseline length of about $9000 \mathrm{~km}$ for the submm-VLBI, and already has a research facility, Summit Camp, which is operated by CH2M Hill]. Polar Services (CPS) for the U.S. National Science Foundation (NSF). The Summit Camp, therefore, meets all four criteria.

The Greenland Summit Camp is located at 72.57 $\mathrm{N}$ latitude and $38.46 \mathrm{~W}$ longitude, at an altitude of $3200 \mathrm{~m}$. The temperature is very low, with winter temperatures between $-30^{\circ} \mathrm{C}$ and $-60^{\circ} \mathrm{C}$, and summer temperatures between $0^{\circ} \mathrm{C}$ and $-30^{\circ} \mathrm{C}$ (Laursen 2010; Martin-Cocher et al. 2014). Due to the combination of the high altitude and the low temperature, very low opacity is expected. Furthermore, the NSF is currently funding the Integrated Characterization of Energy, Clouds, Atmospheric state, and Precipitation (ICECAPS) project at the Summit Camp, which is using active and passive ground-based remote sensors, including two radiometers that observe 16 frequencies from 22.2 $\mathrm{GHz}$ to $150.0 \mathrm{GHz}$, to provide the first complete description of cloud properties above this site (Shupe et al. 2013). They determine the annual cycle of PWV using the radiometer observations at the central frequencies of $23.8,31.4,90$, and $150 \mathrm{GHz}$ as well as from radiosondes that are launched twice daily by ICECAPS technicians. We, therefore, decided to put a $225 \mathrm{GHz}$ tipping radiometer at this site to measure the atmospheric opacity conditions for possible submm VLBI operations (see the next section for the reason to choose the measurement frequency).

Our group is in the process of deploying a $12 \mathrm{~m}$ diameter submm telescope to Greenland. Overall explanations of this project, the Greenland Telescope (GLT) project, are described in Inoue et al. (2014) for submm-VLBI science and technical details, and in Hirashita et al. (2016) for single-dish science cases. In addition, more detailed information about antennas, receivers, and software for the GLT project are in Raffin et al. (2016), Grimes et al. (2014), and Patel et al. (2016), respectively.

\section{MEASUREMENT AND DATA REDUCTION}

\footnotetext{
1 https://www.ch2m.com/
}

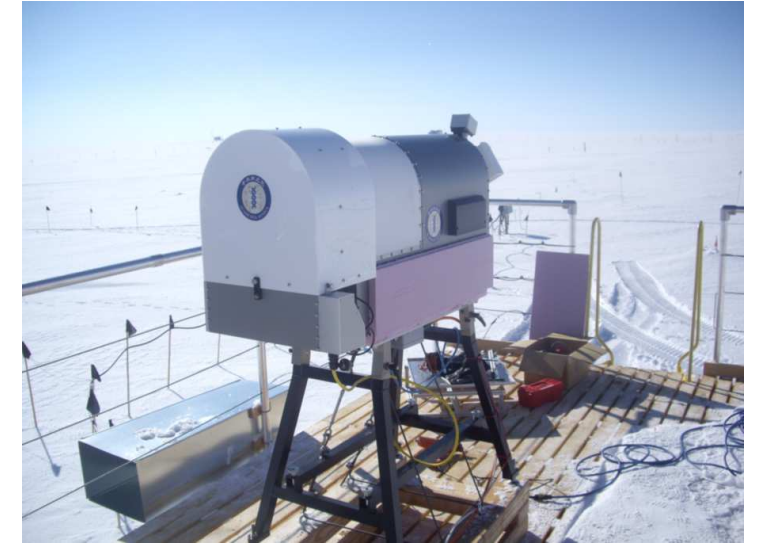

FIG. 1.- The $225 \mathrm{GHz}$ tipping radiometer located on the roof of the Mobile Science Facitily (MSF) at the Greenland Summit Camp.

For the opacity measurement at the summit of the Greenland ice sheet, we procured a $225 \mathrm{GHz}$ tipping radiometer, RPG-225 Radiometer, from Radiometer Physics GmbH (RPG). This instrument has the ability to operate in very cold environments, and indeed some units are operating at Arctic and Antarctic areas (two radiometers operating as part of ICECAPS mentioned above are also from RPG). The reason for the choice of this operating frequency is that there are many site survey results from all over the world, including the current submm telescope sites, such as the summit of Mauna Kea, the ALMA (Chajnantor and Pampa la Bola) site, and South Pole. The radiometer has an uncooled double side band heterodyne receiver with a bandwidth of $1 \mathrm{GHz}$. A tipping paraboloid mirror, which can rotate $360^{\circ}$ with its half power beam width of 0.5 , is installed in front of the feed horn. A Gortex window covers $\pm 90^{\circ}$ from zenith to allow sampling of the sky signal, and a black body target, whose temperature is monitored by a thermometer, is located at the bottom (i.e., $180^{\circ}$ from zenith). A $140 \mathrm{~W}$ blower is located below the window and provides heating to prevent ice formation and accumulation on the window.

We obtained the radiometer in the autumn of 2010 , deployed it on the roof of our institute in Taipei, and conducted functional and gain stability tests. We then moved the radiometer to the summit of Mauna Kea, Hawaii, in the end of 2010, to check the consistency with the $225 \mathrm{GHz}$ tipping radiometer at the Caltech Submillimeter Observatory (CSO). We put our radiometer near the CSO with the same tipping direction, measured the opacity for about 2 weeks, and confirmed that the results were consistent with each other (linear regression coefficient $=1.04)$. After this, we moved the radiometer to the Polar Environment Atmospheric Research Laboratory (PEARL), located on a ridge at an altitude of $610 \mathrm{~m}$ at $80^{\circ} 05 \mathrm{~N}$ and $86^{\circ} .42 \mathrm{~W}, 15 \mathrm{~km}$ away from the Eureka weather station on Ellesmere Island, Canada. We measured the atmospheric opacity for 3 months between late winter to early spring, and the results are reported in Asada et al. (2012) and Matsushita et al. (2013). After this measurement, we moved the radiometer to the Greenland Summit Camp. The radiometer was installed on the roof of the Mobile Science Facility (MSF; Shupe et al. 2013), which was built by CPS in support of the ICECAPS project. The roof deck is at about $3 \mathrm{~m}$ 


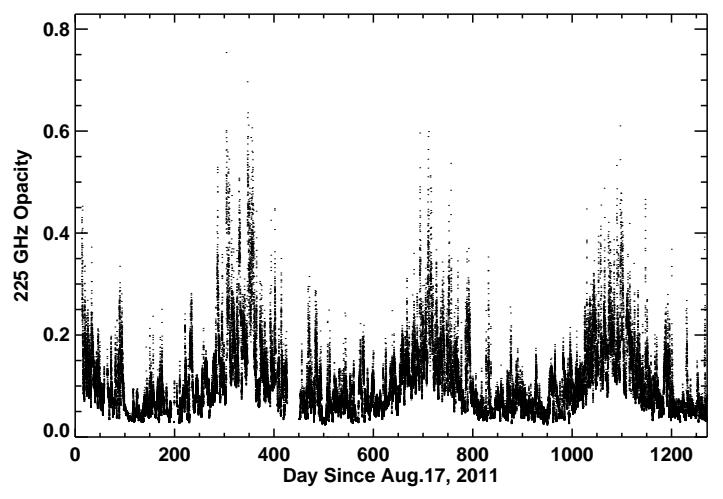

FIG. 2.- Time variation plot of $225 \mathrm{GHz}$ opacity at the summit of Greenland ice sheet. The measurement has been started from Aug. 17, 2011, which is defined as the day 1 in this diagram.

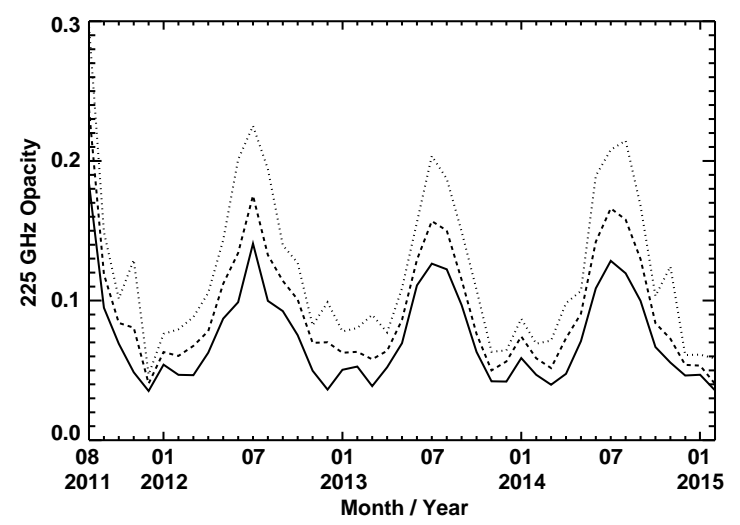

FIG. 3.- Monthly quartile variation of $225 \mathrm{GHz}$ opacity. Solid, dashed, and dotted lines are $25 \%, 50 \%$, and $75 \%$ quartiles, respectively. The first and the last months of this measurement, namely Aug. 2011 and Feb. 2015, have fewer data than the other months, so that the statistical significance is low.

above the snow surface (Fig. 1). The measurement was started from August 17th, 2011, and data were collected until February 12th, 2015. We present here the data for these $\sim 3.5$ years.

To measure the atmospheric opacity, we adopt the tipping method; we observe five angles from horizon $\left(90^{\circ}\right.$ $=$ zenith, $42^{\circ}, 30^{\circ}, 24^{\circ}$, and 19.2 , which corresponds to $\sec (z)$ of $1.0,1.5,2.0,2.5$, and 3.0) with 4 second integration at each angle. We scan the mirror from south to north, namely observe the five angles in both the southern and the northern sky (for the measurements at Greenland). The black body target in the bottom of the radiometer is observed before and after the scan for the gain calibration. The total duration of a tipping measurement is 75 seconds, and each tipping is performed every 10 minutes. Between the tipping measurements, the mirror is pointed toward the zenith and the sky data are recorded every 1 second. The output voltages, together with the mirror position, the black body target temperature, and other monitoring data, are recorded every 1 second into the hard disk of the host computer in binary format. The raw binary data are downloaded from Greenland to Taiwan regularly via internet.

The tipping data have been reduced with the typical data reduction method for tipping measurements (e.g., Matsuo et al. 1998). Since the MSF is a mobile facility

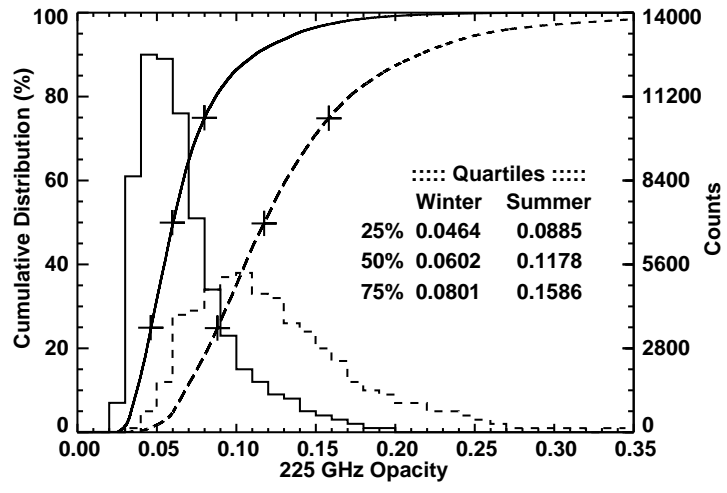

FIG. 4.- Cumulative distribution plots and histograms of 225 $\mathrm{GHz}$ opacity in winter (solid lines) and summer (dashed lines). The vertical axis on the left-hand side is for the cumulative distribution plots, and that on the right-hand side is for the histograms. Crosses on the cumulative distribution plots are the opacity quartiles of each season. The quartile for winter and summer are also listed in the figure.

on the snow, it can shake due to wind or human activities inside the facility, so that the radiometer tipping angle may be affected. In addition, the leveling accuracy for the radiometer is limited, so that the radiometer may also have a small constant tilt. We assume that the opacity is the same between the southern and northern skies, and the small difference in opacities derived from the southern and northern sky tippings is considered as the result of the tilt of MSF and/or the radiometer. Based on this assumption, we calculate the tilt angle and correct for it when deriving the opacity. In case the difference of the opacities between the southern and northern skies is large (i.e., when the tilt angle is calculated to be larger than two degrees; this value is also to allow some tolerance for the different opacities between the northern and southern sky), we judge this is due to real opacity differences, and we flag the data.

\section{RESULTS AND DISCUSSIONS}

\subsection{Time and Seasonal Variations}

Fig. 2 displays the measured time variation of the 225 $\mathrm{GHz}$ opacity for the 3.5-year period at the Greenland Summit Camp. It is clear that there is a seasonal variation; opacity in winter is low, but high in summer. Fig. 3 shows the monthly quartile variations (solid, dashed, and dotted lines are $25 \%, 50 \%$, and $75 \%$ quartiles, respectively). December and March tend to have the best opacity conditions, and July tends to have the worst. Note that the first and the last months of this measurement (i.e., Aug. 2011 and Feb. 2015) have fewer data than the other months, so the statistical significance is lower. In both diagrams, there is no significant annual difference.

\subsection{Cumulative Distribution and Histogram}

We also made cumulative distribution plots and histograms of the measured $225 \mathrm{GHz}$ opacity while separating the seasons into winter and summer (Fig. (4). Here we define winter as between the beginning of November and the end of April (solid line plots), and summer as May through October (dashed line plots). The quartiles for each season are $0.046,0.060$, and 0.080 for $25 \%, 50 \%$, and $75 \%$, respectively, in winter, and $0.089,0.118$, and 0.159 for summer (see also the crosses and the values in the figure). It is obvious that the opacity in winter is 
TABLE 1

Comparison of $225 \mathrm{GHz}$ opacity QuARtiles BetWEen threE SITES.

\begin{tabular}{ccccc}
\hline \hline Site & Season & & Quartiles \\
& & $25 \%$ & $50 \%$ & $75 \%$ \\
\hline GL Summit Camp & Winter & 0.046 & 0.060 & 0.080 \\
& Summer & 0.089 & 0.118 & 0.159 \\
ALMA & Winter & 0.035 & 0.050 & 0.080 \\
& Summer & 0.071 & 0.131 & 0.261 \\
South Pole & Winter & 0.041 & 0.048 & 0.057 \\
& Summer & 0.050 & 0.062 & 0.076 \\
\hline
\end{tabular}

about half of that in summer at all the quartiles. The histograms show that the opacities of 0.04 and 0.10 are the opacities that occur most often in winter and summer, respectively.

We then compared the opacity quartiles using our 3.5-year period statistics with those at the ALMA and the South Pole sites, which are well-established sites for submm observations. The $225 \mathrm{GHz}$ opacity data for the ALMA site have been obtained from Radford \& Chamberlin (2000) and Radford (2011), whose measurements have been made between April 1995 and April 2006 ( 11 years), and that for the South Pole site from Chamberlin \& Ballv (1994, 1995) measured between January and December 1992 (1 year). Since both the ALMA and the South Pole sites are located in the southern hemisphere, we define winter as between the beginning of May through the end of October, and summer as November through April. The calculated quartiles for these three sites are listed in Table 1.

For winter, the ALMA site is the best at the $25 \%$ quartile, South Pole is next, and Greenland Summit Camp is the worst, but only by $\sim 0.005(\sim 12 \%)$ difference in opacity between each site. At the $50 \%$ quartile, the ALMA and South Pole sites are almost the same, and the opacity is about 0.01 (about 25\%) worse at Greenland Summit Camp. At the $75 \%$ quartile, South Pole is the best ( $40 \%$ better) and the opacity is the same between the ALMA and the Greenland sites.

For summer, South Pole is the best for the 25\% quartile, ALMA is the next, and Greenland is the worst with $\sim 0.02$ difference in opacity between each site. At the $50 \%$ and $75 \%$ quartiles, South Pole is again the best, but Greenland is next and the ALMA site is the worst. The South Pole site is about twice better in opacity as compared with the Greenland site, and the ALMA site is significantly worse than these two sites.

We note here that there are many studies that compare the opacity conditions at the summit of Mauna Kea, which is also a well-established submm site, with those at the ALMA and South Pole sites; opacity quartiles of Mauna Kea are $\sim 50 \%$ higher than those of the ALMA site (Matsushita et al. 1999; Radford \& Chamberlin 2000; Radford 2011; Radford \& Peterson 2016).

In summary, the South Pole site has little seasonal differences in opacity over the annual cycle, with a factor of two difference between seasons. On the other hand, the ALMA site has large variations between winter and summer, with significantly worse conditions in summer, known as the Bolivian Winter around February and March. The opacity conditions at the Greenland Summit Camp are roughly intermediate between the South
Pole and the ALMA sites.

\subsection{Diurnal Variation}

The top row of Fig. 5 shows the diurnal opacity variations in winter (left column) and summer (right column) at the Greenland Summit Camp. Solid, dashed, and dotted lines are $25 \%, 50 \%$, and $75 \%$ of the hourly quartiles. It is obvious that there is no diurnal variation in both seasons. This can be easily explained by the polar conditions; only nighttime in winter and daytime in summer. It is also clear that the opacity in winter is half of that in summer, as mentioned in the cumulative distribution plot (Fig. 4) above.

The middle and bottom rows of Fig. 5 show the diurnal variation at the ALMA and the South Pole sites, respectively. It is obvious that there is a clear diurnal variation in the ALMA data, which is naturally explained by the mid-latitude conditions, leading to a strong diurnal cycle compared to polar regions, while the South Pole data are very similar to the Greenland Summit Camp (i.e., no diurnal variation).

For winter, the South Pole site is always the best at all quartiles. Opacities at the 25\% and 50\% quartiles at the ALMA site are almost always statistically better than those at the Greenland Summit Camp, but for the $75 \%$ quartile, the daytime opacity is statistically better at the Greenland Summit Camp than at the ALMA site due to the diurnal variation.

For summer, the South Pole site is again always the best at all quartiles. For opacities at the $25 \%$ and $50 \%$ quartiles, the Greenland Summit Camp is always better between $\sim 12$ and $\sim 20$ hour than the ALMA site (i.e., daytime at the ALMA site) due to no diurnal variation. For opacities at the $75 \%$ quartile, the Greenland Summit Camp is always better than the ALMA site.

\subsection{Duration of Opacity Lower than Certain Values}

We then calculated time durations of opacity conditions continuously lower than certain values. We focused on opacities lower than $0.05,0.04$, and 0.03 , which are excellent opacity conditions that only occur in $\lesssim 30 \%$ of the winter season at the Greenland Summit Camp. The resultant histograms for the Greenland Summit Camp are presented in Fig. 6(a). Since the tipping measurements are done every 10 minutes as mentioned in Sect. 3, the lower limit of the measurements are located at 0.17 hour. The counts (vertical axis) are normalized with the number of annual data points, assuming one data point takes 10 minutes (Sect. 3). We intentionally cut the count limit to 70 in the plot for presentation purpose.

For opacity less than 0.05 or 0.04 at the Greenland Summit Camp, there were several occasions for which more than 100 hours were continuously showing opacities lower than those values, and there are many occasions that continued for more than 10 hours or several hours. The counts for durations between 1 hour and 20 hours are almost the same, and that for durations between 20 hours to several tens of hours are roughly half of the shorter duration. For the opacity less than 0.03 , there were several occasions when the opacity was continuously low for more than 10 hours of time, and more occasions exist for durations of more than an hour. This is obviously due to the polar conditions (no diurnal 

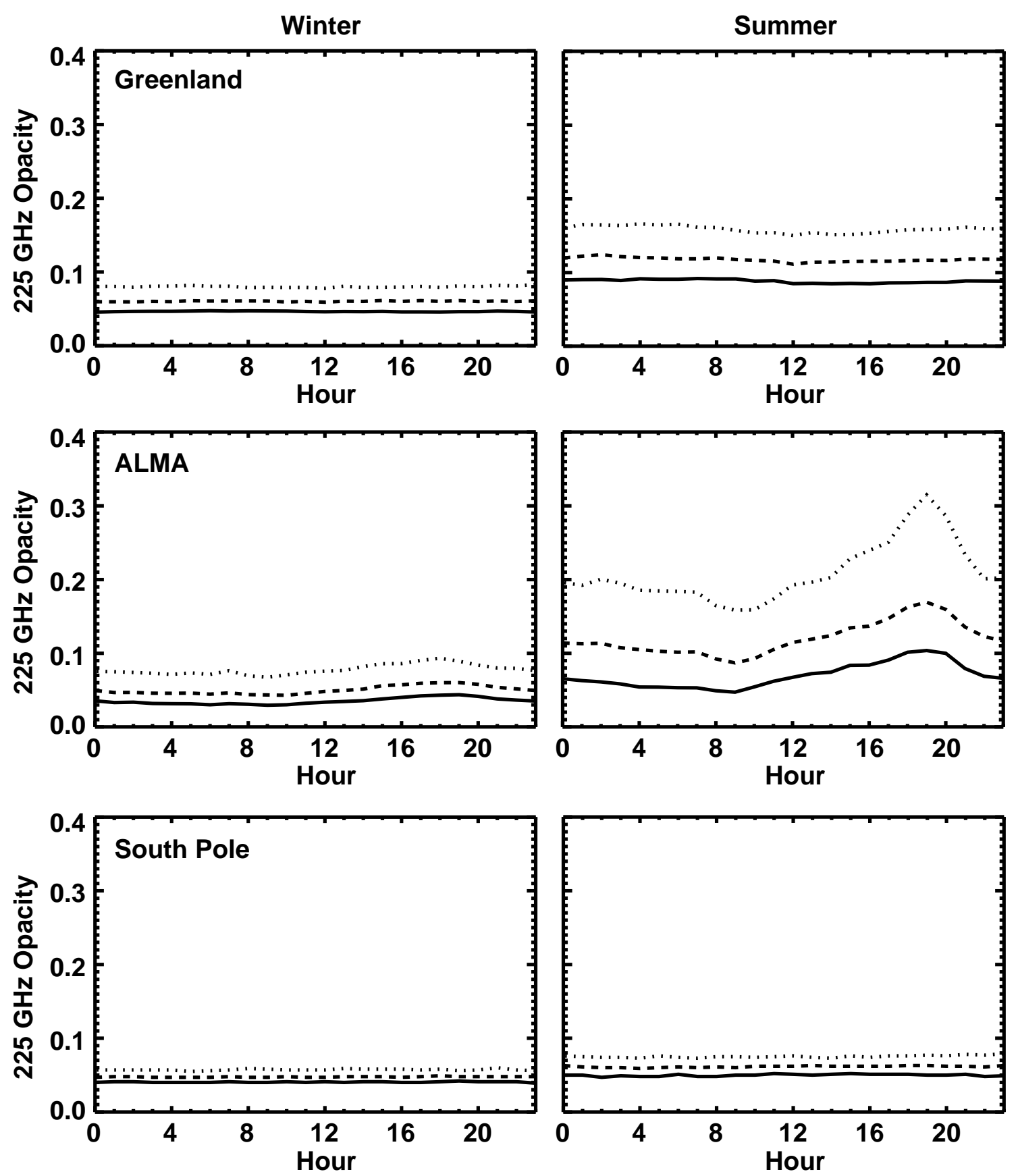

FIG. 5.- Diurnal $225 \mathrm{GHz}$ opacity in winter (left column) and in summer (right column) for the Greenland Summit Camp (top row), the ALMA site (middle row), and the South Pole site (bottom row). Solid, dashed, and dotted lines are $25 \%, 50 \%$, and $75 \%$ of the hourly quartiles.

variation) as mentioned above, and it is very difficult to achieve similar values at other submillimeter sites that are not at polar regions, such as in the Hawaii (Mauna Kea) or Northern Chile (ALMA) site.

Indeed, we also calculated the time duration of opacity conditions for the ALMA site under the same opacity conditions as the Greenland Summit Camp (Fig. 6b), and the difference is obvious. At the ALMA site, there are many occasions for the opacity less than $0.05,0.04$, and even 0.03 continues for up to 20 hours, but the occasions for the duration longer than 20 hours are significantly smaller, about one-fifth, than the shorter dura- tions. The duration longer than 100 hours has only been recorded for opacity lower than 0.05 , and never happened for opacities lower than 0.04 or 0.03 in the 11 yr long data.

For the South Pole site (Fig. 6r), the duration is obviously shorter than that of Greenland Summit Camp. There are many occasions for the opacity less than 0.05 , which continues up to 10 hours, but there are significantly fewer occasions for the duration longer than 10 hours. Similar to the ALMA site, the duration longer than 100 hours has only been recorded in opacity lower than 0.05, and never happened for opacity lower than 
TABLE 2

ATMOSPHERIC OPACITY COUNTS PER YEAR OVER SPECIFIC TIME INTERVALS.

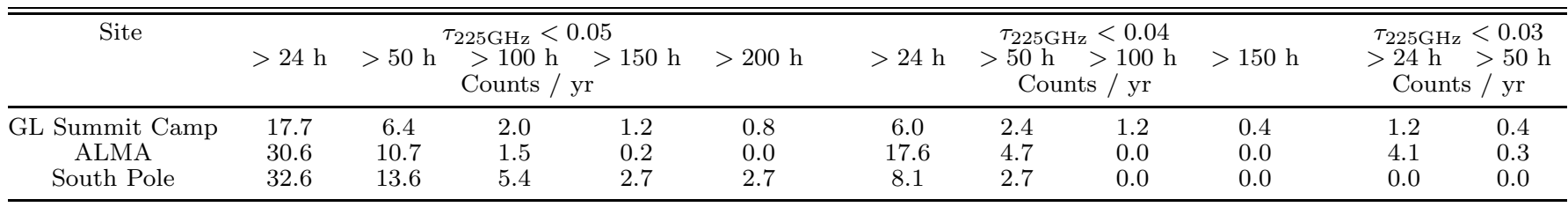
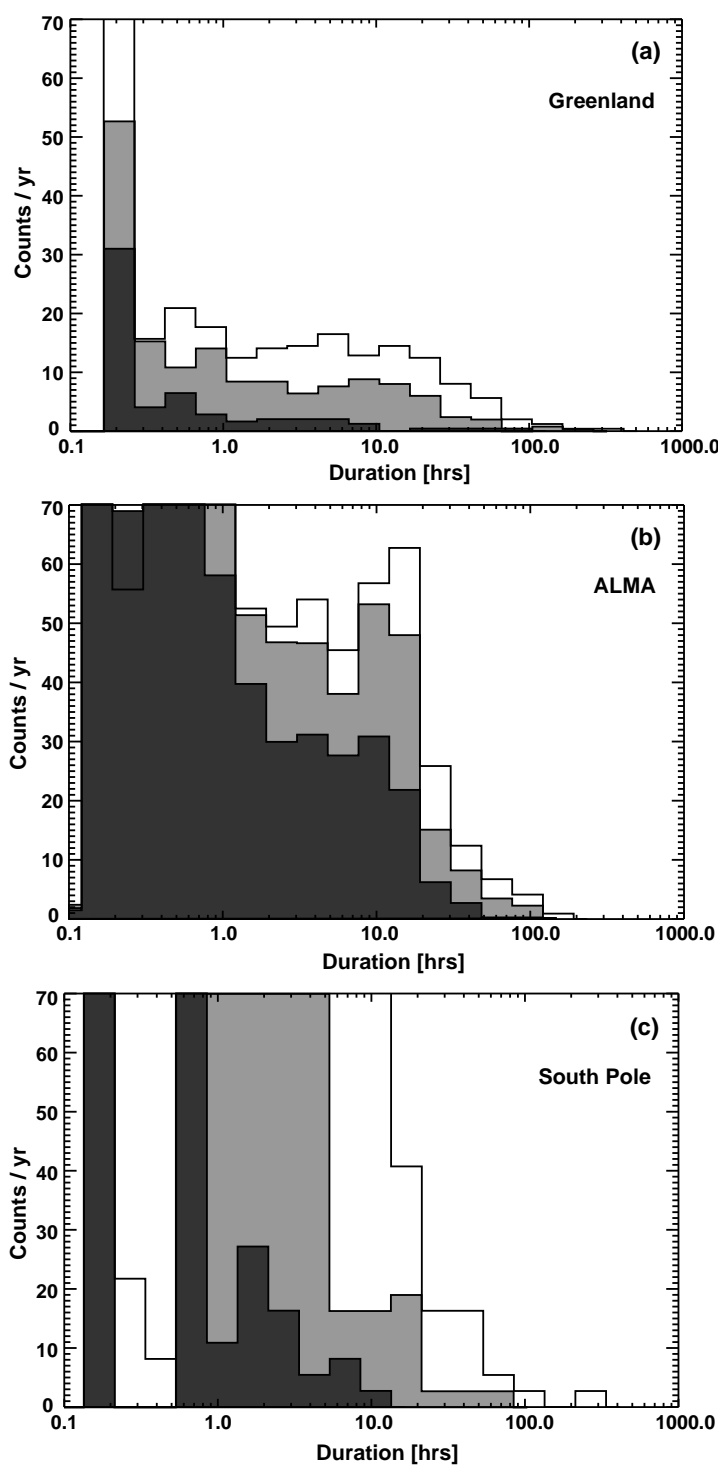

FiG. 6.- Histogram of time duration for opacity continuously lower than 0.05 (white), 0.04 (light grey), and 0.03 (dark grey) for (a) the Greenland Summit Camp, (b) the ALMA site, and (c) the South Pole site. Vertical axis is counts per year.

0.04 or 0.03 . For the opacity lower than 0.03 , the duration is only up to about 10 hours, and has never been longer. Although the South Pole data are taken only for a year and the opacity statistics are better than for the Greenland Summit Camp (Sect. 4.2, 4.3), good opacity duration time is shorter.

Table 2 shows that low opacity conditions continue for more than 24, 50, 100, 150, and 200 hours at the Greenland Summit Camp, ALMA, and the South Pole. The ALMA and South Pole sites have better atmospheric
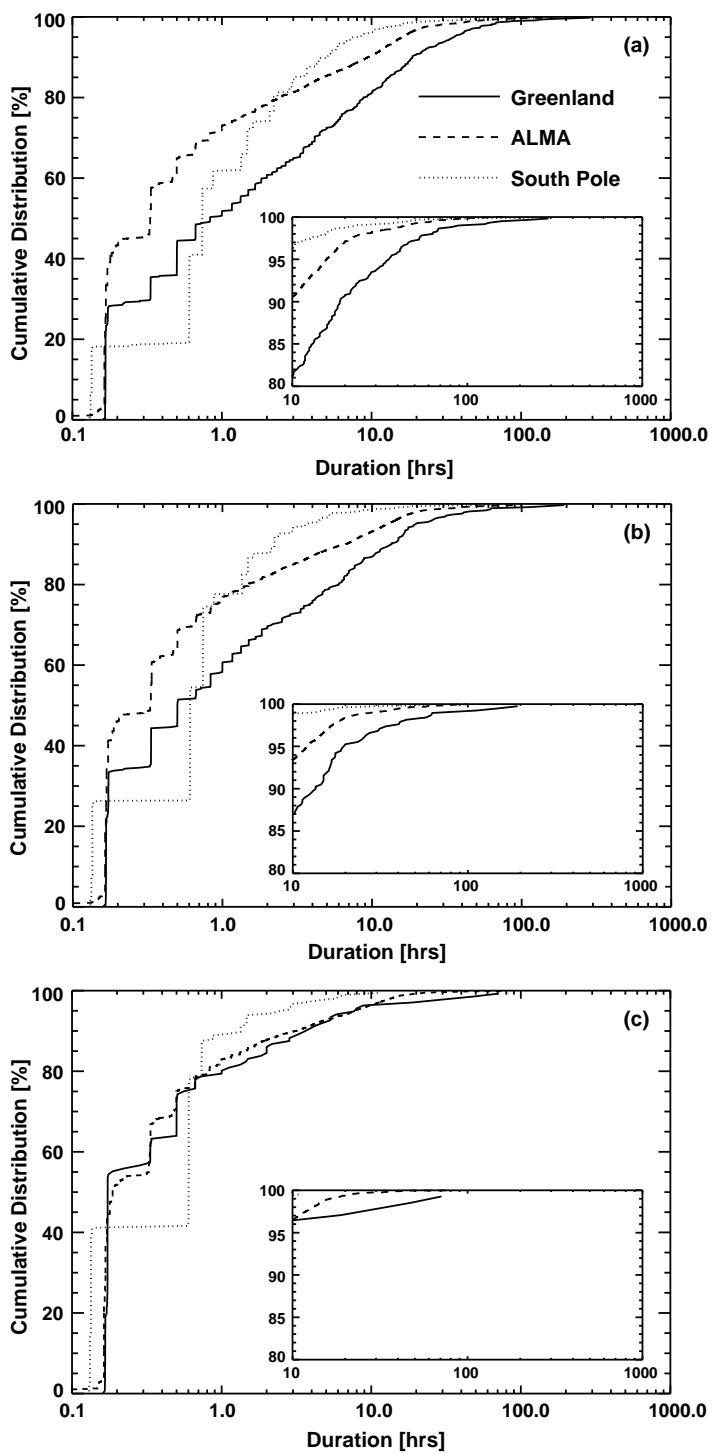

FIG. 7.- Cumulative distribution plots of time duration for opacity continuously lower than (a) 0.05, (b) 0.04 , and (c) 0.03 for the Greenland Summit Camp (solid lines), the ALMA site (dashed lines), and the South Pole site (dotted lines). Each plot also displays an inset that is a zoomed plot of the cumulative distribution between $80 \%$ and $100 \%$ and the time duration between 10 hours and 1000 hours.

opacities over time durations longer than 24 hours and 50 hours at opacity conditions lower than 0.05 and 0.04 , respectively. But for time durations longer than 100 hours at opacity conditions lower than 0.04, the Greenland Summit Camp clearly has lower atmospheric opacities.

We also made a table to show the statistics of low opacity conditions continues for more than 24, 50, 100, 150, and 200 hours in unit of counts per year (Table 2). The 
aforementioned statements are quantitatively shown in this table; the ALMA and South Pole sites have better statistics at time duration longer than 24 hours and 50 hours at opacity conditions lower than 0.05 and 0.04 . But for the time duration longer than 100 hours at opacity conditions lower than 0.04, the Greenland Summit Camp clearly has better statistics.

These results are also clearly seen in Fig. 7 , which compares the cumulative distributions of the time durations for the three sites discussed above. Figs. Z(a) and (b) show the cumulative distributions of the time durations of opacities less than 0.05 and 0.04 , respectively, and it is clear that the Greenland Summit Camp (solid line) has a long tail toward the long duration of more than a hundred hours. The ALMA site always exhibits higher cumulative distributions than that of the Greenland Summit Camp, and reaches $100 \%$ around several tens of hours. The South Pole site shows the steepest cumulative distribution, and reaches $100 \%$ around a few tens of hours, much shorter than the other two sites. The cumulative distributions of the time durations of opacity less than 0.03 (Fig. 7F) display very similar distribution between the Greenland and the ALMA site, but the Greenland Summit Camp shows a long tail up to several tens of hours. Again, the South Pole site is much shorter, only up to ten hours.

In summary, for the low opacity duration, the Greenland Summit Camp is the best site to have continuous low opacity conditions. Since the variation of opacity is directly related to the variation of sky temperature (background), these long stable opacity conditions will be a significant advantage for astronomical observations, which need unusually stable sky background, such as $\mathrm{THz}$ observations or wide-field submillimeter continuum observations.

\section{ESTIMATION OF OPACITIES AND PWVS AT OTHER FREQUENCIES}

Using our $225 \mathrm{GHz}$ opacity data and the radiative transfer program "am" (Paine 2014), we estimated the atmospheric transmission spectra between $0 \mathrm{GHz}$ and $1600 \mathrm{GHz}$ in both winter and summer at the Greenland Summit Camp. First, from the NASA ModernEra Retrospective analysis for Research and Applications, Version 2 (Rienecker et al. 2011; Molod et al.|2015, MERRA-2) reanalysis data interpolated to the location of the Greenland Summit Camp, we computed various percentiles of the temperature, $\mathrm{H}_{2} \mathrm{O}$ mixing ratio, and $\mathrm{O}_{3}$ mixing ratio at each MERRA pressure level, for the winter and summer months covering the same period as the radiometer measurements. The am model files were then constructed such that each percentile model used the corresponding percentile temperature and $\mathrm{H}_{2} \mathrm{O}$ profile, whereas all models used the $50 \%$ quartile $\mathrm{O}_{3}$ profile. Here we assumed that temperature and water vapor should be highly correlated, such that it is physically meaningful to associate a percentile profile with the corresponding percentile statistics on each level. On the other hand, $\mathrm{O}_{3}$ is relatively uncorrelated with either temperature or water vapor, so that it makes sense to simply use the median profile.

For each set of MERRA-2 percentile profiles, we found a scaling factor on the tropospheric part of the $\mathrm{H}_{2} \mathrm{O}$ profile, which reproduced the corresponding $225 \mathrm{GHz}$ opac-
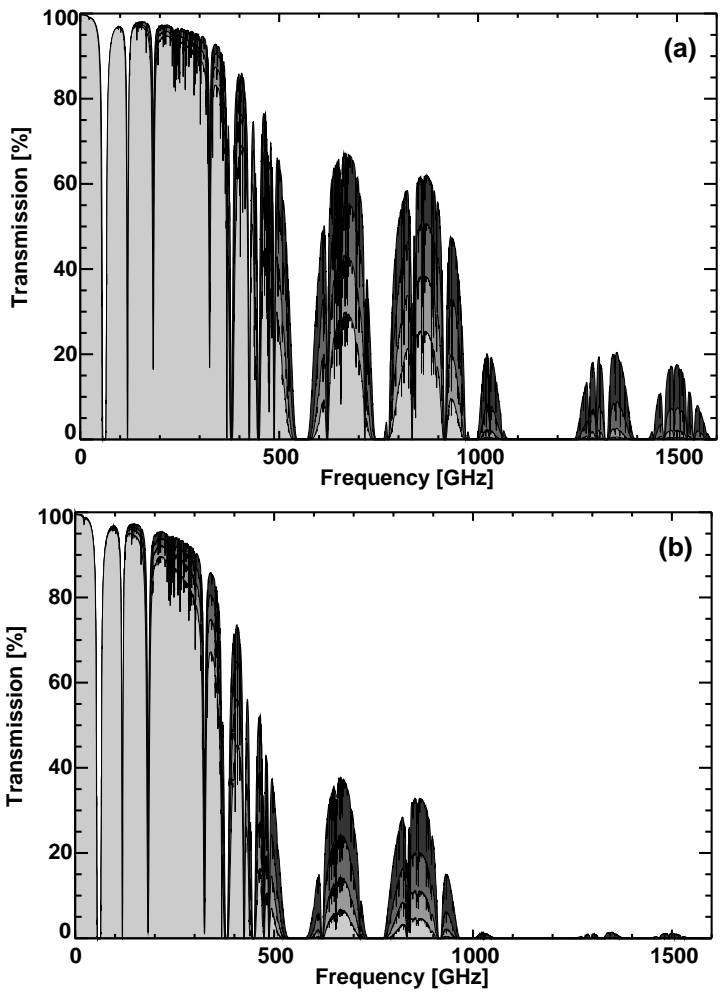

FIG. 8.- Estimated atmospheric transmission spectra at the Greenland Summit Camp in (a) winter and (b) summer seasons. Spectra at $2 \%, 10 \%, 25 \%$, and $50 \%$ opacity conditions are plotted in greyscale with darker to lighter grey.

ity percentiles from our measurements. The scale factor on the median MERRA $\mathrm{H}_{2} \mathrm{O}$ profile to match the median $225 \mathrm{GHz}$ opacity was 1.09 in winter and 1.10 in summer, indicating a dry bias of approximately $10 \%$ for MERRA-2 relative to our measurements. From a radiative transfer model using the percentile profiles scaled to our measurements, we then estimated the corresponding percentile atmospheric transmission spectra.

The estimated atmospheric transmission spectra for the $2 \%, 10 \%, 25 \%$, and $50 \%$ opacity conditions in winter season are plotted in Fig. 8(a). Note that these opacity conditions correspond to the $225 \mathrm{GHz}$ opacity of $0.031,0.037,0.046$, and 0.060 , respectively. These spectra suggest that it is possible to observe astronomical sources with little atmospheric attenuation (opacities $<0.5)$ most of the winter time at the Greenland Summit Camp for frequencies lower than $450 \mathrm{GHz}$, and half of the time for frequencies between $450 \mathrm{GHz}$ and $1000 \mathrm{GHz}$ with opacities $<1.2$. For the $\mathrm{THz}$ windows $(1035 \mathrm{GHz}$, $1350 \mathrm{GHz}$, and $1500 \mathrm{GHz}$ ), $10 \%$ of the winter time will have an atmospheric transmission of more than $10 \%$.

The estimated atmospheric transmission spectra for the same opacity percentiles as above, but in summer season are plotted in Fig. 8(b). Note that these opacity conditions correspond to the $225 \mathrm{GHz}$ opacity of 0.052 , $0.068,0.088$, and 0.118 , respectively. These spectra suggest that it is possible to observe astronomical sources with little atmospheric attenuation (opacities $<0.5$ ) most of the summer time for frequencies below the 380 $\mathrm{GHz}$ water vapor line, and more than half of the time for the $450 \mathrm{GHz}$ atmospheric window with opacities $<1$. For the windows between $450 \mathrm{GHz}$ and $1000 \mathrm{GHz}, 25 \%$ 
TABLE 3

Calculated linear correlation Between $225 \mathrm{GHz}$ and SUBMM ATMOSPHERIC WINDOW OPACITIES AT THE GREENLAND SUMMIT CAMP.

\begin{tabular}{rccc}
\hline \hline Frequency & Coefficient & Offset & $\begin{array}{c}\text { Difference from } \\
\text { the ALMA site }\end{array}$ \\
\hline $345 \mathrm{GHz}$ & 3.80 & -0.032 & $4 \%$ \\
$410 \mathrm{GHz}$ & 7.34 & -0.064 & $-3 \%$ \\
$492 \mathrm{GHz}$ & 26.4 & -0.31 & $12 \%$ \\
$675 \mathrm{GHz}$ & 27.7 & -0.45 & $24 \%$ \\
$875 \mathrm{GHz}$ & 31.0 & -0.48 & $28 \%$ \\
$937 \mathrm{GHz}$ & 55.5 & -0.96 & $26 \%$ \\
$1035 \mathrm{GHz}$ & 140 & -2.7 & $14 \%$ \\
$1350 \mathrm{GHz}$ & 129 & -2.4 & $12 \%$ \\
$1500 \mathrm{GHz}$ & 130 & -2.3 & $29 \%$ \\
\hline
\end{tabular}

of the summer time will have an atmospheric transmission of more than $10 \%$. The $\mathrm{THz}$ windows are totally opaque in summer.

We also calculated the relationships between $225 \mathrm{GHz}$ opacity and other submillimeter atmospheric window opacities using the above estimates. This is useful for estimating the opacities at higher frequencies from the observations of the opacity at $225 \mathrm{GHz}$. Relations between two opacities turned out to be all linear, and therefore the linear coefficients and offsets have been calculated. The calculated values are in Table 3. Opacities at the $492 \mathrm{GHz}, 675 \mathrm{GHz}$, and $875 \mathrm{GHz}$ windows are about 25 - 30 times larger than the $225 \mathrm{GHz}$ opacity, and those at the $\mathrm{THz}$ windows are about $130-140$ times larger.

These values can be compared with the measurement results at the ALMA site (Matsushita et al. 1999, 2000): For the $345 \mathrm{GHz}$ and $410 \mathrm{GHz}$ windows, there are only a few \% differences between the Greenland Summit Camp and the ALMA site, but for higher frequencies, the Greenland Summit Camp is about $10-15 \%$ worse than the ALMA site for the $492 \mathrm{GHz}, 1035 \mathrm{GHz}$, and 1350 $\mathrm{GHz}$ windows, and about $25 \%$ worse for the $675 \mathrm{GHz}$, $875 \mathrm{GHz}, 937 \mathrm{GHz}$, and $1500 \mathrm{GHz}$ windows (Table 3 ). This is due to the altitude difference. The altitude of the Greenland Summit Camp is only $3200 \mathrm{~m}$, much lower than that of the ALMA site at $5000 \mathrm{~m}$, and this difference increases the opacity in the pressure-broadened wings of saturated $\mathrm{H}_{2} \mathrm{O}$ lines at high frequencies. In addition, the lower altitude introduces larger dry air continuum absorption at the Greenland Summit Camp; the dry air continuum absorption increases at higher frequencies, up to the middle of the $\mathrm{N}_{2}-\mathrm{N}_{2}$ collision-induced absorption band near 3 THz (Pardo et al. 2001a, b; Paine 2014).

The "am" program also estimates the PWVs together with the transmission spectra, and we present those in Table 4 Using these values, it is possible to derive the relation between the $225 \mathrm{GHz}$ opacity and PWV at the Greenland Summit Camp, which turned out to be

$$
\tau_{225 \mathrm{GHz}}=0.048 \times \mathrm{PWV}[\mathrm{mm}]+0.022,
$$

where $\tau_{225 \mathrm{GHz}}$ is the $225 \mathrm{GHz}$ opacity. With this equation, together with the relationship between the $225 \mathrm{GHz}$ opacity and that of other frequencies (Table 3), it is also possible to compare various atmospheric window opacities and PWV.

The 3-year (2008 - 2010) PWV statistics and the atmospheric transmission spectra of the summit of Greenland have also been derived by Tremblin et al. (2012) using
TABLE 4

ESTIMATED PWV FOR WINTER AND SUMMER SEASONS.

\begin{tabular}{rcccc}
\hline \hline \multirow{2}{*}{ Percentile } & \multicolumn{2}{c}{ Winter } & \multicolumn{2}{c}{ Summer } \\
& Opacity & {$[\mathrm{mm}]$} & $\begin{array}{c}225 \mathrm{GHz} \\
\text { Opacity }\end{array}$ & $\begin{array}{c}\text { PWV } \\
{[\mathrm{mm}]}\end{array}$ \\
\hline $2 \%$ & 0.0311 & 0.186 & 0.0516 & 0.623 \\
$10 \%$ & 0.0375 & 0.321 & 0.0679 & 0.965 \\
$25 \%$ & 0.0464 & 0.506 & 0.0885 & 1.396 \\
$50 \%$ & 0.0602 & 0.790 & 0.1178 & 2.054 \\
$75 \%$ & 0.0801 & 1.205 & 0.1586 & 2.942 \\
\hline
\end{tabular}

the water vapor product from the Infrared Atmospheric Sounding Interferometer (IASI) instrument on the Meteorological Operation (MetOp)-A satellite. They found a median annual PWV of $0.94 \mathrm{~mm}$ over the summit of Greenland. Our radiometer-derived median PWV for the 3-year subset of our data from October 2011 to September 2014 is $1.28 \mathrm{~mm}$. To connect these two periods, we note that the MERRA-2 median PWV for January 2008 - December 2010 is $1.27 \mathrm{~mm}$, whereas for October 2011 - September 2014 it is $1.16 \mathrm{~mm}$. If we assume the MERRA-2 dry bias of approximately $10 \%$ is consistent between these periods, then the implied median PWV for $2008-2010$ is $1.40 \mathrm{~mm}$. This would suggest that the PWV for Summit in Tremblin et al. (2012) is systematically lower than the actual PWV by approximately $33 \%$.

There are two reasons why a single satellite data set could produce significantly biased PWV statistics. First, although IASI is able to retrieve surface temperature, the water vapor retrieval accuracy in the lower troposphere necessarily suffers from a lack of thermal contrast with the surface (Wulfmeyer et al. 2015). The second reason is temporal sampling bias. Typically satellite sounders are in sun-synchronous orbits that pass over a given point on the Earth's surface at the same pair of local times each day; these are 9:30 and 21:30 in the case of IASI (Hilton et al. 2012). In comparison, a reanalysis such as MERRA-2 can be expected to produce more realistic PWV statistics because it assimilates multiple satellite, surface, and upper air measurements using a model with realistic dynamics.

\section{SUMMARY}

We present the 3.5-yr monitoring of the $225 \mathrm{GHz}$ opacity at the Summit of the Greenland ice sheet using a tipping radiometer.

Opacity variations clearly show a seasonal variation between winter $(\approx$ nighttime $)$ and summer $(\approx$ daytime $)$, but no diurnal variation, with the opacity in winter being about half of that in summer. This is similar to the opacity variations at the South Pole site due to the polar conditions, but the absolute opacity value is about $10 \%$ $30 \%$ higher than that of the South Pole site in winter, and about double in summer. In contrast, the ALMA site shows clear seasonal and diurnal variations due to mid-latitude conditions; the opacity at the ALMA site is up to $20 \%$ better than at the Greenland site in winter, but up to $40 \%$ worse in summer.

The estimated atmospheric transmission spectra suggest that most of the winter time is useable for astronomical observations at frequencies lower than $450 \mathrm{GHz}$, half of the time is useable for frequencies between $450 \mathrm{GHz}$ and $1000 \mathrm{GHz}$, and $10 \%$ of the time is useable for the $\mathrm{THz}$ 
atmospheric windows. Most of summer time is useable at frequencies lower than $380 \mathrm{GHz}$, and half of the summer time is useful at the $450 \mathrm{GHz}$ atmospheric window. The linear correlations between $225 \mathrm{GHz}$ and submillimeter atmospheric window opacities are derived, and opacities at the $492 \mathrm{GHz}, 675 \mathrm{GHz}$, and $875 \mathrm{GHz}$ windows are about $25-30$ times larger, while those at $\mathrm{THz}$ windows are about $130-140$ times larger than the opacity at 225 $\mathrm{GHz}$. These opacities are up to $25 \%$ higher than those at the ALMA site, which is due to the altitude difference between the Greenland Summit Camp (3200 m) and the ALMA site $(5000 \mathrm{~m})$.

The biggest advantage of the opacity conditions at the Greenland Summit Camp is the long time durations of low opacity. At the Greenland Summit Camp, opacities lower than 0.04 or 0.05 can continue for more than a hundred hours occasionally, and opacities lower than 0.03 can continue for more than several tens of hours in some cases. In case of opacity lower than 0.04 , the Greenland
Summit Camp is the only site that can continue this condition for more than one hundred hours, indicating that the Greenland site is suitable for observations that need stable opacity conditions for a long time.

We would like to thank CPS for providing liquid nitrogen yearly, which was used for the calibration of the radiometer's detector, and to the ICECAPS technicians who assisted us in a timely fashion when needed. We also would like to thank Simon J. E. Radford for providing us with the opacity data of the ALMA and the South Pole sites. The MERRA-2 data used in this study have been provided by the Global Modeling and Assimilation Office (GMAO) at NASA Goddard Space Flight Center. We are also grateful to the anonymous referee for helpful comments. SM is supported by the National Science Council (NSC) and the Ministry of Science and Technology (MoST) of Taiwan, NSC 100-2112-M-001-006-MY3 and MoST 103-2112-M-001-032-MY3.

\section{REFERENCES}

Asada, K., Martin-Cocher, P. L., Chen, C.-P., et al. 2012, Proc. SPIE, 8444, 84441J

Bower, G. C., Goss, W. M., Falcke, H., Backer, D. C., \& Lithwick, Y. 2006, ApJ, 648, L127

Chamberlin, R. A. \& Bally, J. 1994, Appl. Opt., 33, 1095

Chamberlin, R. A. \& Bally, J. 1995, Int. J. IR MM Waves, 16, 907

Doeleman, S. S., Weintroub, J., Rogers, A. E. E., et al. 2008, Nature, 455, 78

Doeleman, S. S., Fish, V. L., Schenck, D. E., et al. 2012, Science, 338,355

Grimes, P. K., Asada, K., Blundell, R., et al. 2014, Proc. SPIE, 9153, 91531V

Hilton, F., Armante, R., August, T., et al. 2012, Bull. American Met. Soc., 93, 347

Hirashita, H., Koch, P. M., Matsushita, S., et al. 2016, PASJ, 68, 1

Inoue, M., Algaba-Marcos, J. C., Asada, K., et al. 2014, Radio Science, 49, 564

Laursen, E. V. 2010, DMI Technical Report No.10-09

Martin-Cocher, P. L., Asada, K., Matsushita, S., Chen, M.-T., Ho, P. T. P., \& Chen, C.-P. 2014, Proc. SPIE, 9147, 91473N

Matsuo, H., Sakamoto, A., \& Matsushita, S. 1998, PASJ, 50, 359

Matsushita, S., Chen, M.-T., Martin-Cocher, P., et al., 2013, IAU Symp., 288, 204

Matsushita, S., Matsuo, H., Pardo, J. R., \& Radford, S. J. E. 1999, PASJ, 51, 603
Matsushita, S., Matsuo, H., Sakamoto, A., \& Pardo, J. R. 2000, Proc. SPIE, 4015, 378

Molod, A., Takacs, L., Suarez, M., \& Bacmeister, J. 2015, Geoscie. Model Dev., 8, 1339

Paine, S. 2014, SMA Technical Memo, 152

Pardo, J. R., Cernicharo, J., \& Serabyn, E. 2001a, IEEE Trans. on Antennas and Propagation, 49, 1683

Pardo, J. R., Serabyn, E., \& Cernicharo, J. 2001b, J. Quant. Spec. Radiat. Transf., 68, 419

Patel, N. A., Nishioka, H., \& Huang C.-W. L. 2016, Proc. SPIE, 9913, 991308

Radford, S. J. E. 2011, Rev. Mex. Astronomía y Astrofísica (Serie de Conferencias), 41, 87

Radford, S. J. E. \& Chamberlin, R. A. 2000, ALMA Memo, 334

Radford, S. J. E. \& Peterson, J. B. 2016, PASP, 128, 965, 075001

Raffin P., Ho, P. T. P., Asada, K., et al. 2016, Proc. SPIE, 9906, 99060U

Rienecker, M. M., Suarez, M. J., Gelaro, R., et al. 2011, J. Climate, 24, 3624

Shen, Z.-Q., Lo, K. Y., Liang, M.-C., Ho, P. T. P., \& Zhao, J.-H. 2005, Nature, 438, 62

Shupe, M. D., Turner, D. D., Walden, V. P., et al. 2013, Bull. American Met. Soc., 94, 169

Tremblin, P., Schneider, N., Minier, V., Durand, G. Al., \& Urban, J. 2012, A\&A, 548, A65

Wulfmeyer, V., Hardesty, R. M., Turner, D. D., et al. 2015,

Reviews of Geophysics, 53, 819 